clange, and $I$ have now to mention it in connexion with the latter, the intensity of the light being dependent upon the density of the substance and the elevation of temperature, but the state of any body when incondescent is not well understood. The inmense difference of temperature required for the ionition or different substances is easily seen by some simple experiments. If I hold a piece of platinum wire in the flame of a spirit lamp, it exhibits a greater brilliancy of light than the flame itself, and that arises from the greater density of the wire; at the same time it must be understood, that there is no one substance which may not become luminous on a proper elevation of the temperature. The definition of flame, as given by $\mathrm{Sir}$ Isaac Newton, was that which accords with our present knowledge of it, namely, "that it is ignited vapour." Now, here you may observe a flame arising from a jet of hydrogen gas, which is scarcely visible, which shows us that flame may exist without any solid substance,-it is therefore ignited vapour; and that it possesses a considerable degree of heat, may be seen from its power of heating platinum wire to a great state of incondescence. But generally speaking, in the flame of a candle or lamp, it is the charcoal liberated by the decomposition of the material employed, which gives a body to the flame, which is the chief substance of ignition. If you apply a cold substance over a flame; or especially if we hold a wire gauze over a flame, we extinguish a portion of the flame, because we coul it, the wire gauze being an excellent conductor and radiator of heat, and that the cooled gaseous matter passes through, may be seen by again lighting it upon its upper surface. The tlame of phosphorus is, as you know, exceedingly bright, which depends merely on the circumstance, that the flame of phosphorus is a solid substance, whereas the combustion of matter generally is in a state of vapour. The experiment was made of holding a wire gauze over the flame of phosphorus, and sufficient phosphorus passed through the wire to support combustion again on the opposite side when kindled. The discovery of these facts respecting the nature and properties of flame, led Sir H. Davy to apply them to the construction of that valuable contrivance, the miners' safety lamp, which will be explained when we have to consider the properties of carburetted hydrogen gas.

In the next Lecture, Mr. Brande will commence the description of the simple supporters of combustion.

\section{FOREIGN DEPARTMENT.}

ON THE MODUS OPERANDI OF ALOES, AND ITS MEDICAI PROPERTIES.

BY DR. WEDEKTND.

Ir is well accertained that the purgative effect of aloes does not take place till eight and sometimes twelve hours after its exhibition. Individuals in whom the secretion of bile is increased with the greatest facility, are those most powerfully acted upon by aloes. An excitement of the whole system, with quickness of the pulse, disagreeable heat of the abdomen, dryness of the mouth, succeeds the administration of this remedy. These effects are increased when the medicine is continued during se. veral days, and if exhibited in very strong doses, it sometimes gives rise to serious hæmorrhages.

From experiments made on persons in health, and observations on persons in sickness, it has been found that a purgative which acts speedily, as, for instance, a mixture composed of a laxative infusion and sulphate of soda given at one time, with two or four grains of aloes, does not act differently than if exhibited alone. But aloes given two hours before the mixture, does not commence to operate till the effect of the latter has ceased, and the evacuations in second purging differ from those in the first, as well as in appearance as in smell. If, on the contrary, the aloes be given from six to eight hours before the mixture, the effects of the two remedies coincide, and the evacuations are generally very copious.

If the stools produced by the aloes are not watery, but bilious, fæculent, and have a specific smell, it is not owing to a special action on the large intestines, as authors have generally stated. The author of this paper had several opportunities of trying this remedy in icterus; and as long as the stools were white or grey, aloes did not purge even when exhibited in large doses; but the purgative effect immediately supervened, as soon as the frecal matter commenced to contain bile, a proof that the presence of bile in the intestinal canal is a necessary condition of the purgative effect of the aloes. If the aloes were given in too large doses, a bilious diarrhœa generally followed.

It is, then, useless to give aloes with neutral salts, and other salts which act promptly, unless it is desired to promote the secretion of bile; and under such circum. stances, the aloes should be given same hours before the other medicine. The pan- 
creatic and hepatic secretions will be still more increased by the addition of calomel.

It appears, then, that the primitive effect of the aloes is on the liver, that this organ is excited by it as the salivary glands are by mercury, or the kidneys by cantharides; that its purgative effect does not depend, as that of many other catbartics, on an increased secretion from the intestinal canal, and an immediate excitement of the muscular fibres of the intestine; but the aloes is first absorbed, then carried into the circution, and afterwards secreted by the liver, the action of which it increases.

Dr. Wedekind thinks, that the use of aloes is principally indicated when the secretion of bile is scanty, in constipation, from atony of the colon and rectum, and in icterus, which may be attributed to atony of the liver. This medicine should be employed with much caution in irritable persons. In cases of icterus, attended with a spasmodic or inflammatory state of the liver, obstructions of the liver with dropsy, as well as in persons affected with biliary calculi, and those disposed to hæmorrhoids, its use is decidedly improper.

\section{INCUBATED EGG.}

The various vital processes of nutrition and formation, which are carried on in the foetus of the mammalia while in its mother's body, and by means of the most intimate connexion with the parent, are effected in the incubated chick by its own powers, quite independently of the mother, and without any extraneous substance, except that of the atmospheric air, and a certain degree of warmth. The egg is covered, within the shell, by a white and firm membrane (membrana albuminis), which contains no blood-vessels. The two layers of this membrane, which in other parts adhere closely to each other, leave at the large end a space which is filled with atmospheric air. This membrane includes the two whites of the egg, each of which is surrounded by a deli. cate membrane. The external of these is the most fluid and transparent, the inner one thicker and more opaque ; they may be separated in eggs which are boiled hard.

The internal white surrounds the yolk, which is contained in a peculiar membrane called the yolk-bag. From each end of this proceeds a white knotty body, which terminates in a flocculent extremity in the albumen. These are called the chalaze or grandines. Leveille distinguishes a third white, and considers the chalazæ as absorbing ressels floating in it, and destined to absorb it as well as the inner albumen, and mix them with the yolk during incubation. A small round milk-white spot, called the tread of the cock (cicatricula or macula) is formed on the surface of the yolk-bag. It is surrounded by one or more whitish conzentric circles (halones or circelli), the use of which, as well as that of the cicatricula itself, and of the chalaze, is not yet ascertained. We now proceed to notice the wonderful successive changes which go on during the incubation of the egg ; and the metamorphoses which are observed both in the general form of the chick, and in particular viscera. The periods of these changes will be set down from the hen, as affording the most familiar example. It will be best to give, first, a cursory chronological view of the whole process, and then to make a few remarks on some of the most important parts of the subject. A small shining spot of an elongated form, with rounded extremities, but narrowest in the middle, is perceived at the end of the first day, not in nor upon the cicatricula, but very near that part on the yolk-bag (nidus pulli, colliquamentum, areole pellucida). This may be said to appear before-hand, as the abode of the chick which is to follow. No trace of the latter can be discerned before the beginning of the second day, and then it has an incurvated form resembling a gelatinous filament, with large extremities very closely sur. rounded by the amnion, which, at first, can scarcely be elistinguished from it. About this time the halones enlarge their circles, but they soon after disappear entirely, as well as the cicatricula.

The first appearance of red blood is dis. cerned on the surface of the yolk-bag, towards the end of the second day. A series of points is observed, which form groores, and these closing, constitute vessels, the trunks of which become connected to the chick.' The vascular surface itself is called figura venosa, or arca vasculosa, and the vessel by which its margin is defined, vena termi. nalis. The trunk of all the veins joins the vena portæ, while the arteries, which ramify on the yolk.bag, arise from the mesenteric artery of the chick. On the com. mencement of the third day, the newly. formed heat (the primary organ of the cir. culating process which now commences) is discerned by means of its triple pulsation, and constitutes a three-fold punctum saliens. Some parts of the incubated chicken are destined to undergo successive alterations in their form; and this holds good of the heart in particular. In its first formation it resembles a tortuous canal, and consists of three dilations lying close together, and arranged in a triangle. One of these, which is properly the right, is then the common auricle; the other is the only ventricle, but afterwards the left; and the third is the dilatated part of the aorta (bulbus aorte). About the same time, the spine, which was originally extended in a straight line, becomes 